\title{
A Bayesian Approach to in vivo Kidney Ultrasound Contour Detection Using Markov Random Fields
}

\author{
Marcos Martín and Carlos Alberola* \\ ETSI Telecomunicación. Universidad de Valladolid \\ Cra. Cementerio s/n, 47011 Valladolid, Spain \\ \{marcma, caralb\}@tel.uva.es
}

\begin{abstract}
Automatic detection of structures in medical images is of great importance for the implementation of tools that can obtain accurate measurements for an eventual diagnosis. In this paper, a new method for the creation of such tools is presented. We focus on in vivo kidney ultrasound, a target in which classical methods fail due to the inherent difficulty of such an imaging modality and organ. The proposed method operates on every slice by detecting kidney contours under a probabilistic Bayesian framework. We make use of Markov Random Fields ideas to model the problem and find the solution. A computer easy-to-use interface to the model is also presented.
\end{abstract}

\section{Introduction}

Neonatal hydronefrosis is a disease of great relevance in the fetus and newborn children. It consists of an enlargement of the renal pelvis and calyces. Its early diagnosis is a common task thanks to the use of echography, both during the pregnancy or in the newborn and is becoming the more frequent prenatal urologic diagnosis.

Echographical analyses permit determining whether this or other urological diseases are present; the current inspection process is as follows: after scanning an adequate slice, the specialist manually adjusts — helped with cursors - an ellipse to the guessed external boundary of the kidney. The system approximates the kidney volume as the volume of the ellipsoid generated by rotating the sketched ellipse about its main axis. The pelvis volume is determined similarly. From those approximations, the specialist reaches a diagnosis using tabulated tendency data.

An automatic or semiautomatic segmentation tool will be, in our opinion, of valuable importance, not only for the determination of the kidney and pelvis contours by means of a more accurate method than the one described above, but also for automatically propagating those contours to the rest of the slices of the volume scanned. With such an approach, the volume estimates are expected

\footnotetext{
* The authors acknowledge the Comisión Interministerial de Ciencia y Tecnología for research grants 1FD97-0881 and TIC2001-3808-C02-02, and Junta de Castilla y León for research grant VA91/01.
} 
to be better in terms of accuracy, and also in relaxing the need of the specialist to find a slice with sufficient quality.

In this paper, we describe how such a tool can be developed. After reviewing the literature about this and related topics, we highlight the deficiencies of the methods previously proposed. This will be done in Sect. 2. In Sect. 3we describe our proposal. Sect. 4 is dedicated to show how the computer application is used. It will be obvious from the description that no technical knowledge is needed to make use of the application. Finally, Sect. 5 describes some experiments carried out on real echograchical data to validate the method here proposed.

\section{State of the Art}

Several approaches are described in the literature to improve measurements of the volume enclosed within a kidney with respect to that obtained by the ellipse method. In [15] the authors describe a wholly manual segmentation method. In that approach, the contours are sketched manually in a slice-by-slice basis on the ultrasound data acquired from an in vivo kidney. From those contours, the volume is calculated by means of voxel counting. Such a measurement is proven to be better that the one obtained with the ellipse method. In [12] the authors propose an in vitro semiautomatic segmentation method; kidney contours are fairly obvious due to the organ-liquid interface in which the kidney is submerged.

Classical approaches to image segmentation are only valid under extremely controlled situations. In most cases, high level techniques which exploit prior knowledge about the shape of the structures to be segmented out are the only feasible solution. Active contours (snakes) are one of the most successful approaches [9]. The solution contour is determined by finding optimal contours in a neighborhood of the initial guess. The optimality of the solution in a real setting together with the dependence of the initial guess is matter of discussion.

One alternative to the optimization methods proposed in $[9$ is to reformulate the problem in a Bayesian probabilistic framework and make use of Markov Random Fields (MRFs) 6]. In that framework, prior distributions will model our knowledge about the contours and data-driven likelihood terms will describe the image statistics related to the contour in search. The maximum a posteriori (MAP) estimation under the MRF models give rise to the optimal contour.

Several proposals that make use of this philosophy have already been reported. In [4] an automatic algorithm has been developed for the detection of the left ventricular (LV) cavity boundaries in sequential 2D echocardiograms. This work is pioneer in defining a contour model in polar coordinates as shown in Fig. 11(a). From a given center $\left(C_{x}, C_{y}\right)$ the plane is discretized in polar coordinates which give rise to $J$ contour points $\rho_{j}$ at equispaced angular positions $\theta_{j}$ - the radii-. Each contour point $\rho_{j}$ can only be one out of $K$ values $r_{k}$. In the approach [4] a region of interest is defined beginning with an ellipse adjusted by using the Hough transform. The field is defined by means of image borders, contour smoothness, maximal volume and temporal continuity. The optimal contour 


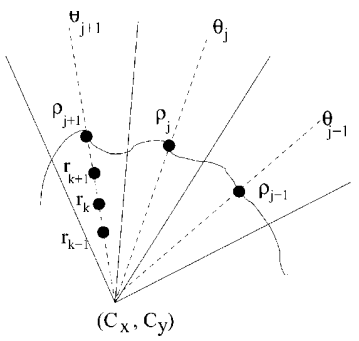

(a)

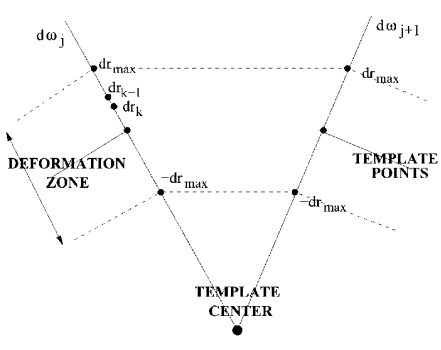

(b)

Fig. 1. (a) Classical contour model. (b) Proposed contour deformation.

is found by using the Simulated Annealing (SA) algorithm [6] on the induced MRF. The parameters are determined experimentally.

The approach [5] is very similar to , but it is developed for infrared images. The prior model is rather heuristic and the likelihood is based on the image gradient. The model weights are fixed by means of a proof-and-error method.

A further step is [3], in which the model of [4] is modified for the detection of the LV boundaries in angiographic images. The prior model is based on contour smoothness and the likelihood assumes a Gaussian distribution. The approach is Bayesian, they use the Iterated Conditional Modes (ICM) [1] algorithm for optimization, and the parameters are estimated from image data.

In [13] the contour model is represented in Cartesian coordinates, where the number of nodes is random. The optimal contour is given by a Bayesian estimation assuming a fractal measurement as prior model and likelihood based on Gaussian distribution and image gradient. The model is not strictly a MRF; instead, a Markov chain is constructed whose limiting distribution is the MAP estimation - a modified version of SA algorithm is proposed. Several results are presented for LV echocardiograms and brain MRI. The time taken by the algorithm is unacceptable in a clinical setting.

In [2] the authors make use of the results of 34] for the detection of the internal and external boundaries of LV cavity in sequences of echocardiograms. The probabilistic model captures the heart morphology and the physical image generation mechanisms. They assume Rayleigh distribution for the image with data-driven parameters. For the MAP estimation they developed a suboptimal iterative multigrid dynamic programming (IMDP) algorithm. Recently, the model proposed in 2] has been used for segmenting 3D intravascular ultrasonic images [7].

Two previous approaches to our model have already been published. In [10] a sequence of echographies from an in vitro fetus were segmented using active contours with several new energy terms. The optimization approach was based on the relaxation labeling method [8] which provides suboptimal solutions. In [1] the problem of object detection in speckle images was addressed. A regularized maximum likelihood (ML) method was used to determine the contour of the object. A Beta distribution was used to model the image intensity distribution. 
That distribution was compared with the others proposed in the literature 14 and, in addition to its simplicity, results obtained were satisfactory. In both approaches the contour representation was the one shown in Fig. 1(a).

The segmentation problem for in vivo kidney ultrasound volumes is rather involved and must take into account several aspects not dealt with in the above mentioned proposals. The kidney is a soft organ and its form and position may vary with time. The kidney interior is by no means homogeneous due to several distinguishable structures. There is not a clear difference between the kidney and the surrounding tissues. Only some parts of the kidney contour show a significative image gradient. In some scans, the kidney might be partially occluded. All these issues highlight the need of a novel method that may solve the problem.

\section{The Model}

We propose a MRF of deformations with respect to a predefined template of a kidney contour. Specifically, the template is manually or automatically adjusted to the image and then it is smoothly deformed following the outstanding boundaries in the ultrasound image and guided by an empirical speckle distribution model as well. The smoothness constraint is imposed by the range of allowable deformation values and also by the prior distribution, which forces first and second order derivatives smoothness. The final contour is given as the MAP estimation.

\subsection{The Prior Function}

A manually adjusted template $(\boldsymbol{\rho}, \boldsymbol{\theta})$ in polar coordinates is assumed to be available (see Sect. (4). Assume it consists of $J$ points at fixed angles $\theta_{j}$ from a given center. From that template we define a deformation zone as shown in Fig. 团b). The deformation vector will be denoted with $\boldsymbol{d} \boldsymbol{\rho}$. This vector is assumed to be a sample of a prior MRF $\boldsymbol{d} \boldsymbol{\omega}$. Each $d \rho_{j}$ can take on values in the finite set $\boldsymbol{d} \boldsymbol{\Lambda}$. The field of sites will be denoted by $\mathbf{S}=\{1, \ldots, J\}$ and the configuration space with $\boldsymbol{d} \boldsymbol{\Omega}=\boldsymbol{d} \boldsymbol{\Lambda}^{\mathrm{S}}$; therefore, each configuration $\boldsymbol{d} \boldsymbol{\rho}$ belongs to $\boldsymbol{d} \boldsymbol{\Omega}$. The finite set $\boldsymbol{d} \boldsymbol{\Lambda}$ has $K$ equispaced points in the interval $\left[-d r_{\max }, d r_{\max }\right]$.

We have defined a homogeneous and periodic prior neighborhood system $\boldsymbol{\partial}$ which is given by $\boldsymbol{\partial}(j)=\{j-2, j-1, j+1, j+2\}$. The prior MRF distribution $\Pi$ defined for $\boldsymbol{d} \boldsymbol{\omega}$ will be induced by a neighborhood potential $\mathcal{V}$ with two types of functions. The first type is $U_{j}(\boldsymbol{d} \boldsymbol{\rho})=\vartheta_{1} \Psi\left(d \rho_{j-1}-d \rho_{j+1}\right)$; these functions make use of two-site cliques and impose first-order-derivative smoothness. The second type is $V_{j}(\boldsymbol{d} \boldsymbol{\rho})=\vartheta_{2} \Psi\left(d \rho_{j-1}-2 d \rho_{j}+d \rho_{j+1}\right)$. In this case, three-site cliques are used and these functions impose second-order-derivative smoothness [9]. The function $\Psi(x)$ must be monotonic for $x \geq 0$ and even. Prior local field characteristics are

$$
\begin{gathered}
\Pi\left(d \omega_{j}=d \rho_{j} / \boldsymbol{d} \boldsymbol{\omega}_{\boldsymbol{\partial}(j)}=\boldsymbol{d} \boldsymbol{\rho}_{\boldsymbol{\partial}(j)}\right) \\
=\frac{1}{Z_{j}} \exp \left\{-U_{j-1}(\boldsymbol{d} \boldsymbol{\rho})-U_{j+1}(\boldsymbol{d} \boldsymbol{\rho})-V_{j-1}(\boldsymbol{d} \boldsymbol{\rho})-V_{j}(\boldsymbol{d} \boldsymbol{\rho})-V_{j+1}(\boldsymbol{d} \boldsymbol{\rho})\right\}
\end{gathered}
$$

with $Z_{j}$ a normalizing constant. 


\subsection{The Likelihood Function}

The ultrasonic image will be denoted by I. From that image we determine a non-linearly compressed gradient image denoted by $\mathbf{B}$. The probability density function of the data conditioned to the deformation will be

$$
\mathrm{f}(\mathbf{I}, \mathbf{B} / \boldsymbol{d} \boldsymbol{\omega}=\boldsymbol{d} \boldsymbol{\rho}) \propto \prod_{j=1}^{J} \mathrm{f}\left(\mathbf{I}\left(k_{j}, j\right) / d \omega_{j}=d_{k_{j}}\right) \mathrm{f}\left(\mathbf{B}\left(k_{j}, j\right) / d \omega_{j}=d_{k_{j}}\right),
$$

where the indexes $k_{j}$ are chosen to achieve that $\left\{d \rho_{1}, \ldots, d \rho_{J}\right\}=\left\{d r_{k_{1}}, \ldots\right.$, $\left.d r_{k_{J}}\right\} . \mathbf{I}(k, j)$ and $\mathbf{B}(k, j)$ are subimages of $\mathbf{I}$ and $\mathbf{B}$, respectively, whose set of pixels $\boldsymbol{\beta}(k, j)$ are near the contour deformation at angle position $j$ and distance position $k$. We define a log-likelihood function as

$$
L_{\mathbf{B}}(k, j)=-\frac{1}{|\boldsymbol{\beta}(k, j)|} \sum_{(m, n) \in \boldsymbol{\beta}(k, j)} B(m, n) \propto \ln \mathbf{f}\left(\mathbf{B}(k, j) / d \omega_{j}=d r_{k}\right) .
$$

Using a Beta distribution [11] with parameters depending on the pixel positions with respect to the contour deformation we also define the log-likelihood function

$$
\begin{aligned}
L_{\mathbf{I}}(k, j) & =\sum_{(m, n) \in \boldsymbol{\beta}^{i}(k, j)} \frac{\left(1-\alpha_{1}^{i}(j)\right) \ln (I(m, n))+\left(1-\alpha_{2}^{i}(j)\right) \ln (1-I(m, n))}{\left|\boldsymbol{\beta}^{i}(k, j)\right|} \\
& +\sum_{(m, n) \in \boldsymbol{\beta}^{e}(k, j)} \frac{\left(1-\alpha_{1}^{e}(j)\right) \ln (I(n, m))+\left(1-\alpha_{2}^{e}(j)\right) \ln (1-I(m, n))}{\left|\boldsymbol{\beta}^{e}(k, j)\right|} \\
& \propto \ln \mathbf{f}\left(\mathbf{I}(k, j) / d \omega_{j}=d r_{k}\right),
\end{aligned}
$$

where $\boldsymbol{\beta}^{i}(k, j)$ and $\boldsymbol{\beta}^{e}(k, j)$ are partition subsets of $\boldsymbol{\beta}(k, j)$ with the internal and external set of pixels respect to the contour deformation point $j$ at position $k$, respectively. The function $L_{\mathbf{I}}(k, j)$ has $4 J$ Beta shape parameters $\left(\alpha_{1}^{i}(j), \alpha_{2}^{i}(j)\right.$, $\left.\alpha_{1}^{e}(j), \alpha_{2}^{e}(j)\right)$ which can be estimated directly from the ultrasound image I [11].

\subsection{The Posterior Model}

Bayes theorem allows us to write

$$
\mathrm{P}(\boldsymbol{d} \boldsymbol{\omega}=\boldsymbol{d} \boldsymbol{\rho} / \mathbf{I}, \mathbf{B}) \propto \Pi(\boldsymbol{d} \boldsymbol{\omega}=\boldsymbol{d} \boldsymbol{\rho}) \prod_{j=1}^{J} \mathrm{f}\left(\mathbf{I}\left(k_{j}, j\right) / d \omega_{j}=d_{k_{j}}\right) \mathrm{f}\left(\mathbf{B}\left(k_{j}, j\right) / d \omega_{j}=d_{k_{j}}\right),
$$

which is proportional to

$$
\Pi^{p}(\boldsymbol{d} \boldsymbol{\omega}=\boldsymbol{d} \boldsymbol{\rho})=\frac{1}{Z^{p}} \Pi(\boldsymbol{d} \boldsymbol{\omega}=\boldsymbol{d} \boldsymbol{\rho}) \prod_{j=1}^{J} \exp \left(-\vartheta_{3} L_{\mathbf{I}}\left(k_{j}, j\right)-\vartheta_{4} L_{\mathbf{B}}\left(k_{j}, j\right)\right),
$$

where $\Pi^{p}$ is a posterior MRF distribution induced by a posterior neighborhood potential $\mathcal{V}^{p}$ defined on the same $\boldsymbol{\partial}$ and $Z^{p}$ is a normalizing constant. Posterior 
potential functions will be of three types: $U_{j}^{p}(\boldsymbol{d} \boldsymbol{\rho})=U_{j}(\boldsymbol{d} \boldsymbol{\rho}), V_{j}^{p}(\boldsymbol{d} \boldsymbol{\rho})=V_{j}(\boldsymbol{d} \boldsymbol{\rho})$, and $W_{j}^{p}(\boldsymbol{d} \boldsymbol{\rho})=\vartheta_{3} L_{\mathbf{I}}\left(k_{j}, j\right)+\vartheta_{4} L_{\mathbf{B}}\left(k_{j}, j\right)$, with $\boldsymbol{\vartheta}^{p}=\left(\vartheta_{1}, \vartheta_{2}, \vartheta_{3}, \vartheta_{4}\right)$ the vector of posterior parameters. The potential functions $W_{j}^{p}$ are for one-site cliques and impose image restrictions [9]. Posterior local field characteristics are

$$
\begin{gathered}
\Pi^{p}\left(d \omega_{j}=d \rho_{j} / \boldsymbol{d} \boldsymbol{\omega}_{\boldsymbol{\partial}(j)}=\boldsymbol{d} \boldsymbol{\rho}_{\boldsymbol{\partial}(j)}\right) \\
=\frac{1}{Z_{j}^{p}} \exp \left\{-U_{j-1}^{p}(\boldsymbol{d} \boldsymbol{\rho})-U_{j+1}^{p}(\boldsymbol{d} \boldsymbol{\rho})-V_{j-1}^{p}(\boldsymbol{d} \boldsymbol{\rho})-V_{j}^{p}(\boldsymbol{d} \boldsymbol{\rho})-V_{j+1}^{p}(\boldsymbol{d} \boldsymbol{\rho})-W_{j}^{p}(\boldsymbol{d} \boldsymbol{\rho})\right\}
\end{gathered}
$$

with $Z_{j}^{p}$ a normalizing constant. The solution contour is the one that maximizes this posterior, which can be found with the SA algorithm [6].

\section{Running the Application}

The method proposed can be dealt with by means of an easy-to-use computer application; this environment allows the end user to interact with the data, supervise results, manually adjust parameters if desired and so forth.

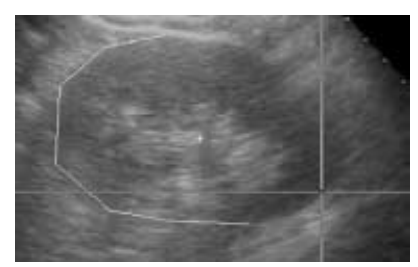

(a)

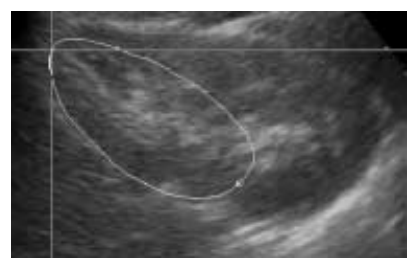

(c)

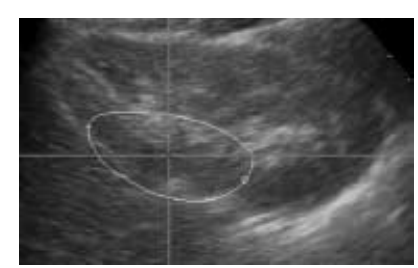

(b)

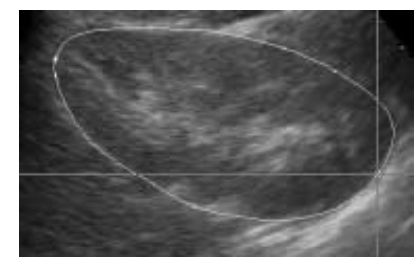

(d)

Fig. 2. (a) A canonical template is drawn. (b)-(d) Template adjustment to the slice.

As previously described, the model needs a template from which deformations start taking place. This model can be created once and stored for future use. Note that the user does not need to draw a template every time a segmentation is needed, but just once. This model could even be built-in; however, we have chosen the former option just to give more flexibility to the end user.

The template definition is very simple: on a chosen slice, the center, together with several points around the kidney contour, are clicked in with the mouse. This set of points is interpolated to get a smooth contour. Fig. 22(a) shows the 
progress of this operation. This is the only information needed to create the polar representation of the template that will be used in the segmentation process.

As far as segmentation is concerned, the procedure is fairly straightforward as well; the user has to choose a candidate slice from which the segmentation process is to be triggered. Now, the application superimposes a normalized version of the stored template (see Fig. 2(b)). Then, the user manually adjusts two hot points in the template to the actual contour of the kidney (see Figs. 2(c)-(d)). At this point, the segmentation procedure described in the paper is launched.

To automatically evolve to further slices, the original template is affinely deformed according to the contour solution of the current slice and superimposed into the consecutive slice, thus, forcing smoothness along the depth coordinate.

\section{Some Experimental Results}

Two experiments will illustrate and validate the segmenting capability of the model proposed. In the first one, we have employed a series of $2 \mathrm{D}$ echographies of an adult healthy kidney. The template is adjusted to slice number 101 (out of 126 ). We have used $J=70$ rays, number of points per ray $K=15$, and the deformation zone consisting of an interval given by $d r_{\max }=20$ pixels. The vector of posterior parameters of the energy function is $\boldsymbol{\vartheta}^{p}=(25,25,5,10)$. The SA algorithm has been run with 250 iterations. Fig. 3.(a) shows slice number 89 and Fig. 3(d) shows the resulting segmented contour. Fig. 3)(b) shows slice number 122 and Fig. 3(e) the corresponding segmented contour.

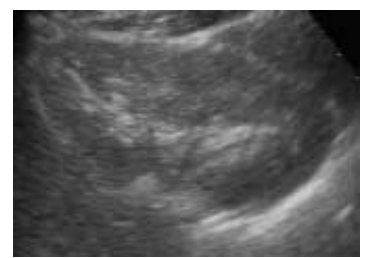

(a)

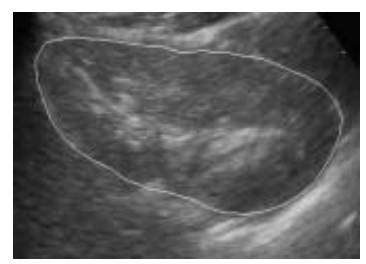

(d)

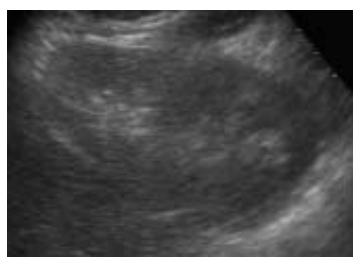

(b)

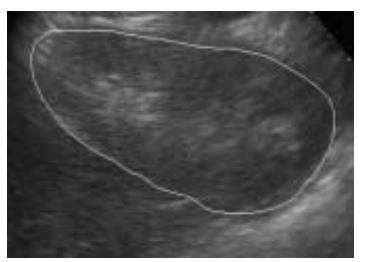

(e)

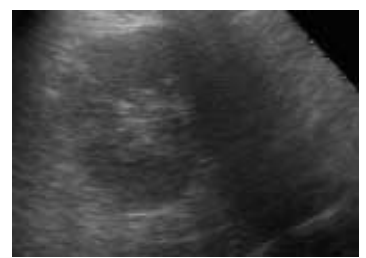

(c)

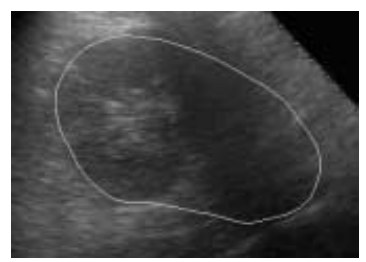

(f)

Fig. 3. (a)-(f) Experimental results.

In the second experiment we have used the data belonging to another adult healthy patient. The template has been adjusted to slice number 30 . The condition of the experiments are as before but for the number of iterations of the SA algorithm, which is 200, and for the vector of posterior parameters of the energy 
function, which are now $\boldsymbol{\vartheta}^{p}=(20,20,7,12)$. Fig. 3)(c) shows slice number 20, in which a severe occlusion makes the kidney contour unclear for visual inspection. Fig. 3(f) shows the segmented contour.

\section{Conclusions and Further Works}

The method here proposed is a step towards an eventual objective procedure that would make diagnosis less dependent of the ecographist skills. Prior knowledge about the average kidney together with empirical knowledge about the echographies have been put together in a probabilistic framework. Segmentation has been therefore posed as an estimation problem for which the existence of an optimal solution is guaranteed.

Several issues are until unaddressed, though. Internal structures of the kidney are still unsegmented. However, once the external contour is found, internal structures are confined in space, which makes a posterior search more tractable.

\section{References}

1. Besag, J.: On the Statistical Analysis of Dirty Pictures (with Discussion). J.Roy. Stat. Soc. B48 (1986) 259-302.

2. Dias, J., Leitao, J.: Wall Position and Thickness Estimation from Sequences of Echocardiographic Images. IEEE TMI 15 (1996) 25-38.

3. Figueiredo, M., Leitao, J.: Bayesian Estimation of Ventricular Contours in Angiographic Images. IEEE TMI 11 (1992) 416-429.

4. Friedland, N., Adam, D.: Ventricular Cavity Boundary Detection from Sequential Ultrasound Images Using Simulated Annealing. IEEE TMI 8 (1989) 344-353.

5. Friedland, N., Rosenfeld, A.: Compact Object Recognition Using Energy-FunctionBased Optimization. IEEE TPAMI 14 (1992) 770-777.

6. Geman, S., Geman, D.: Stochastic Relaxation, Gibbs Distributions and the Bayesian Restoration of Images. IEEE TPAMI 6 (1984) 721-741.

7. Haas, C., et al.: Segmentation of 3D Intravascular Ultrasonic Images Based on a Random Field Model. Ultr. Med. Biol. 26 (2000) 297-306.

8. Hummel, R., Zucker, S.: On the Foundations of Relaxation Labeling Processes. IEEE TPAMI 5 (1983) 267-287.

9. Kass, M., et al.: Snakes: Active Contour Models. I. J. Comp. Vis. 2 (1988) 321-331.

10. Martín, M., et al.: Energy Functions for the Segmentation of Ultrasound Volume Data using Active Rays. Proc. IEEE ICASSP, Istanbul, Turkey (2000) 2274-2277.

11. Martín, M., et al.: Maximum Likelihood Contour Estimation Using Beta-Statistics in Ultrasound Images. Proc. IEEE ISPA, Pula, Croatia (2001) 207-212.

12. Matre, K., et al.: In Vitro Volume Estimation of Kidneys Using 3D Ultrasonography and a Position Sensor. Eur. J. Ultr. 10 (1999) 65-73.

13. Storvik, G.: A Bayesian Approach to Dynamic Contours through Stochastic Sampling and Simulated Annealing. IEEE TPAMI 16 (1994) 976-986.

14. Tuthill, T., et al.: Deviation from Rayleigh Statistics in Ultrasonic Speckle. Ultr. Imag. 10 (1988) 81-89.

15. Yu, C.H., et al.: Fetal Renal Volume in Normal Gestation: A 3D Ultrasound Study, Ultr. Med. Biol. 26 (2000) 1253-1256. 